\title{
A Framework for the Development of New Socio-economic Scenarios for Climate Change Research: Introductory Essay
}

\section{A Forthcoming Special Issue of Climatic Change}

\author{
Nebojsa Nakicenovic • Robert J. Lempert • \\ Anthony C. Janetos
}

Received: 31 January 2013 / Accepted: 9 October 2013 /Published online: 30 January 2014

(C) The Author(s) 2014. This article is published with open access at Springerlink.com

Historically, scenarios have served multiple uses for climate-related research and decisionmaking. Scenarios provide an essential scientific method for exploring possible future climatse change and its impacts. They also help inform consideration of decisions regarding mitigation and adaptation. Over the last several decades, the climate change research community has produced two widely-used sets of scenarios. The development of a new scenario set is now underway. This volume reports on a novel and central component of this effort - the development of shared socio-economic development pathways (SSPs).

Emissions scenarios, a precursor of today's SSPs, have described future emissions of greenhouse gases (GHGs) and other short-lived and long-lived forcings of the physical climate system. Such emissions scenarios have evolved substantially over time, driven in large part by improvements in the integrated assessment models (IAMs) used to create them. Whereas the earliest scientific assessments, such as the first IPCC scientific assessment (IPCC 1990), used a

This article is part of the Special Issue on "A Framework for the Development of New Socio-economic Scenarios for Climate Change Research” edited by Nebojsa Nakicenovic, Robert Lempert, and Anthony Janetos.

N. Nakicenovic $(\square)$

International Institute for Applied Systems Analysis (IIASA), IIASA, Schlossplatz 1, A-2361

Laxenburg, Austria

e-mail: naki@iiasa.ac.at

N. Nakicenovic

e-mail: naki@eeg.tuwien.ac.at

R. J. Lempert ( $\square)$

RAND, Santa Monica CA, USA

e-mail: lempert@rand.org

A. C. Janetos

The Frederick S. Pardee Center for the Study of the Longer-Range Future, Boston University,

Boston, MA 02215, USA

e-mail: ajanetos@bu.edu

N. Nakicenovic

Vienna University of Technology (TU WIEN), TU Wien, Gusshausstrasse 25, A-1010 Vienna, Austria 
very simple $1 \%$ per year increase of atmospheric $\mathrm{CO}_{2}$ concentrations to drive climate models, today's climate modeling community uses internally consistent projections of GHG emissions produced by Integrated Assessment Models (IAM's) that include not only fossil fuel combustion, but explicit representations of changes in land use and the energy system. Such emissions scenarios have become the standard for investigating the robustness of a wide variety of mitigation strategies.

Concurrently, the climate impacts community has used the outputs from General Circulation Models (GCMs) as the raw material for climate scenarios, from which studies of potential impacts have been derived. Such climate scenarios may be taken directly from GCMs, or may be downscaled through a wide variety of methods. These scenarios are essential not only for understanding potential impacts, but also as input for possible decision-making about adaptation to climate change's effects.

In each case, the flow of information has been one-way, from one set of models to another, with little opportunity for feedback among the three primary research communities mitigation researchers, climate modelers, and impacts, adaptation, and vulnerability researchers. Investigations of the assumptions of each community in generating or using scenarios have only rarely been examined; and the opportunities for investigating feedbacks among these three domains even less so.

These previous generations of scenarios for emissions and atmospheric concentrations of greenhouse gases have nonetheless proved enormously influential. The Intergovernmental Panel on Climate change (IPCC) first developed sets of long-term emissions scenarios in 1990 and 1992 (Leggett et al. 1992; Pepper et al. 1992). In particular, the IPCC Special Report on Emissions Scenarios (SRES, Nakicenovic et al. 2000) has been used widely for well over a decade, as the standard for impacts, mitigation and adaptation studies. The SRES process began with a literature review focused on understanding the range of driving forces affecting emissions of GHGs and other radiatively active substances. This review informed the crafting of four narrative storylines describing the main scenario characteristics. These storylines were then used to guide development of initial quantitative scenarios by six leading IAMs. These scenarios were then posted on a public website and announced in scientific journals to solicit feedback. Following this "open process," and IPCC expert and government review, forty quantitative scenarios were then published and posted online.

Some six years ago it became evident, given new knowledge and rapid change in the world's economy and technology, that a new set of scenarios would be required to meet the emerging needs of different scientific communities and the resulting IPCC assessments. In addition, a broad consensus arose across climate change communities that a new approach for developing these scenarios was needed to enhance interactions among the three primary research communities (CM, IAV and IAM) and to adequately address the complexity of the interactions of human decisions about mitigation and adaptation, and about the climate system and climate impacts.

The Aspen Workshop in 2006 (Meehl and Hibbard 2007) initiated this new process and the IPCC Workshop on Scenarios in Noordwijkerhout, Netherlands, in 2007 (Moss et al., 2008, 2010) formulated the basic framework that includes the SSPs that are the focus of this special issue. This new process includes at least two novel features. First, the process began with the specification of a set of four forcing pathways, known as the Representative Concentration Pathways (RCPs). In contrast, previous scenario exercises began with emissions scenarios, then produced scenarios for atmospheric concentrations, which were then given as inputs to the GCMs, whose results could finally be used by the IAV community. From start to finish, this process could take close to a decade. In contrast, this new process works in parallel, with the IAM and GCM modelers both working simultaneously with the new RCPs. Second, this new process has been organized by the 
scientific community according to guidelines set by the IPCC, rather than through the framework of an IPCC Special Report.

The community has already completed development of the RCPs and documented them in a special issue of Climatic Change (van Vuuren et al. 2011). Climate and earth-systems model simulations based on these pathways have been completed and are being analyzed as part of the CMIP5 exercise (Taylor et al. 2012). The RCPs are trajectories of GHG concentrations with radiative forcing endpoints that are widely enough separated for climate modelers to construct a set of experiments to investigate carbon-climate feedbacks and other emerging research questions. They have been developed by four IAMs and as such imply consistent socioeconomic and mitigation assumptions for each of the four RCPs, but these were not reported and will be replaced by new driving forces specified by SSP and shared policy assumptions (SPAs) through the mentioned parallel process. All RCPs implicitly include mitigation actions in their formulation that are sufficient to stabilize radiative forcing at the end of the 21 st century at different levels, including "overshoot" pathways and net "negative" emissions.

These accomplishments suggest initial success towards the goals of more and faster interactions among the various climate research communities. But this new scenario process also poses new challenges. Each of the RCP's implies unspecified assumptions about socioeconomic trajectories - such as the degree of economic development in the world, how it is distributed geographically, the rapidity and eventual level of population growth, and a variety of assumptions about the spread of technologies, all of them driven by market forces. These assumptions rest in part in the structure of the underlying models - do they or don't they include land-use changes interacting with changes in the energy system, for example - and in part in the judgment of the modeling teams that have produced both reference and policy cases. Furthermore, these assumptions have important implications not only for GHG mitigation questions, but also for understanding the potential for adaptation measures. Furthermore, no RCP implies a unique set of socioeconomic assumptions. Numerous socioeconomic pathways could lead to each of the RCPs and similar socioeconomic pathways may lead to different RCPs depending on the adaptation and mitigation assumptions.

This special issue documents the community's efforts to address some of these new challenges. Researchers seek to explore the similarities and differences among the potential pathways consistent with any one RCP. Researchers seek to make the assumptions built into those pathways specific and transparent so their consequences can be better understood. The concept of SSPs emerged to identify, quantify (to the extent possible), and analyze sets of assumptions about ways in which societies may evolve, independently of their decisions about climate change policies. As such the SSPs constitute multiple baseline pathways, which can be combined with studies specifically about climate related policies, both mitigation and adaptation, for new insights into the sensitivity of strategies to underlying socioeconomic trends, as well as to study the interactions of mitigation and adaptation strategies.

Over the past two years, researchers have worked to develop the SSP's. A series of meetings has helped to facilitate progress and synthesize approaches. A joint IPCC-NAS workshop in Washington, DC, in 2010 explored the needs for socioeconomic and environmental futures that could be used with climate scenarios (NRC 2010) and served as a stimulus for a number of proposed frameworks within which such scenarios could be developed (van Vuuren et al. 2012; Kriegler et al. 2012). An IPCC Workshop on Socioeconomic Scenarios held in Berlin in November 2010 brought together researchers from the integrated assessment modeling (IAM); impacts, adaptation and vulnerability (IAV); and climate modeling (CM) communities to consider the task and led to adoption of a unified framework for the development of a small set of Shared Socio-economic Pathways (SSPs) and their use in conjunction with the RCPs and 
associated climate model simulations for the development and assessment of integrated research (IPCC 2012).

Subsequent to the Berlin meeting, a team of authors produced a manuscript documenting an over-arching conceptual framework for the development and use of SSPs (Arnell et al. 2011). This "framework paper" was posted on a public website (www.isp.ucar.edu/socio-economicpathways) and an invitation to submit comments was circulated widely throughout the research community. ${ }^{1}$ Seventeen individuals submitted comments on the paper, totaling 23 pages of critiques. In early November, 2011, a meeting in Boulder, Colorado, was held with the joint purpose of adopting a basic set of narratives for the SSPs and laying out priorities for further activities (O’Neill et al. 2012). The framework paper served as the key background document to this meeting, and many of the presentations were directly relevant to the SSP framework and its implementation.

In essence, the conceptual framework for the development on new scenarios can be described as a three-dimensional matrix with RCPs on one dimension, SSPs, on a second, and SPAs on the third. This simplified picture does not capture possible linkages and feedbacks among RCPs, SSPs and SPAs. For example, an affluent world with high technological capabilities would tend to have both high adaptive and mitigative capabilities and thus result in low climate stabilization pathway. With this structure, SSPs and SPAs jointly specify essential scenario driving forces that with uncertainty, and other possible complications such as interdependence SSPs and SPAs, determine the feasibility or likelihood of a particular RCP being achieved. Different sets of SSPs and SPAs can lead to one particular RCP and the other way around a particular SSP with differing SPAs might lead to alternative RCPs.

Thus, the SSPs and SPAs can be thought of as independent or interdependent scenario components, two axes of a three-dimensional matrix, that jointly determine the RCPs. Each RCP can then be thought of as determined by some joint subset of the SSP and SPA dimensions. For example, stronger policy assumptions may make a given RCP consistent with a range of SSPs that it is not consistent with under weaker policy assumptions. In principle, this conceptual framework allowes a more focused discussion of how feedbacks would complicate the scenario formulation and use by allowing for explicit interaction between SSPs and SPAs: Certain SPAs may be compatible with this SSP but not that one; there may be interactions and feedbacks that link RCPs to SSPs and SPAs through realized climate impacts (e.g., severe climate-change impacts suppress growth, and divert capital away from innovation); and interactions and feedbacks from RCPs to SPAs through either realized or anticipated impacts (e.g., widespread alarm over impacts makes stronger mitigation policies feasible). This special issue aims to document this conceptual framework for the new scenarios as well as provide research and commentary on key related issues. This special issue draws from, but goes beyond papers and presentations given at the meeting on new socioeconomic scenarios held in Boulder in November 2011.

This special issue begins with an essay that sets the stage and is followed by eleven papers divided into four groups. The first group describes the new scenario framework, the second group proposes means to enhance the consistency of scenarios produced by this framework, the third describes research uses of such scenarios, and the fourth proposes new concepts that might enrich the development of these and future scenarios.

\footnotetext{
${ }^{1}$ Invitations to submit comments were circulated widely to personal contacts as well the following email lists: IPCC AR5 WG2 and WG3 authors; the HDGEC and CLIMLIST listservs, and the Population-Environment Research Network.
} 
The stage-setting essay by Ebi et al. (2013) presents the background to development of the new SSP framework, addresses the motivation for initiating this very ambitious and challenging process back in 2006 and describes the process itself underlying the new scenario framework.

The first group of three papers describes the new SSP framework, which aims to guide the development of integrated scenarios combining climate model projections, socioeconomic conditions, and assumptions about climate policies.

The paper by van Vuuren et al. (2013) presents a framework for developing community scenarios for the use in climate research and assessments. It offers a matrix architecture of how the existing RCPs are related to new SSPs based on two main axes: the level of forcing (as represented by the RCPs) and different socio-economic reference pathways. The framework can be used as a tool for classifying new scenarios alongside existing ones and as a guide for developing additional scenarios. This architecture also addresses a key question of current climate research, namely the identification of trade-offs and synergies in terms of risks, costs and other consequences of different adaptation and mitigation strategies.

The second paper of this group by O'Neill et al. (2012) provides a conceptual framework for combining radiative forcing and their associated climate changes from RCPs with alternative pathways of socio-economic development from existing and new SSPs. They characterize SSPs as plausible alternative trends in the evolution of society and ecosystems over a century timescale, in the absence of climate change or climate policies and discuss how particular trends in social, economic, and environmental development could be combined to produce climate outcomes given by RCPs. A comparison to the narratives from the scenarios developed in SRES (Nakicenovic et al. 2000) is used as an illustration of how a starting point for developing SSPs can be defined.

The third paper by Kriegler et al. (2013) presents the concept of shared climate policy assumptions (SPAs) as an important element of the new scenario framework. Shared climate policy assumptions give the mitigation and adaptation measures as they are not specified in the SSPs, and therefore introduce an important third dimension to the scenario matrix architecture. They conclude that a meaningful set of shared climate policy assumptions is useful for grouping individual climate policy analyses and facilitating their comparison. For a given combination of SSP and RCP, both the climate policy costs, including adaptation and mitigation, as well as the residual climate impacts will vary with the climate policy assumptions. Of course elements other than costs and benefits are needed for a full appraisal of policies.

These framework papers highlight a major challenge - how to understandably and transparently merge the SSPs, SPAs and RCPs into useful set of scenarios? The SSP framework presented in the three papers provides a methodological basis and process, but the combinatorics remain daunting. Many SSPs and SPAs with four basic RCPs, with and without climate policies, would lead to a huge number of possible scenarios. On one hand, this represents a great advantage of the framework, which provides a "library" of many scenarios, from global and regional to sectoral, that share some of the basic assumptions. This large set serves the community "building" aspect and could potentially meet the specific needs of the many different potential users. On the other hand, many applications require a succinct and small number of scenarios. Accordingly, the remaining papers in this special issue explore a variety of ways to address these tensions.

The second group of three papers proposes means to enhance the consistency of the new SSPs by comparing them to past scenarios relevant to the SSPs, by using quantitative measures of scenario self-consistency, and by evaluating their underlying assumptions about institutions using insights from the political science and political economy literatures. 
The first paper by van Vuuren and Carter (2013) assesses the past scenarios in the literature with a particular focus on emissions scenarios and explores their relevance for the SSP framework. Important examples, for instance, include the IPCC IS92 and SRES scenarios developed to explore climate change problems and the scenarios of the Millennium Ecosystem Assessment (MA) to explore sustainability issues. They categorize the scenarios in the literature into six different groups on the basis of their storylines (narratives). The main objective is to link in a useful and consistent manner the RCPs and SSPs to the large existing emissions scenarios literature.

In the second paper, Schweizer and O'Neill (2013) use cross-impact balance analysis to evaluate the internal self-consistency of alternative combinations of challenges to adaptation and mitigation. They begin by identifying and prioritizing 13 determinants of such challenges at a globally aggregated scale based on a survey of 25 experts. A cross-impact balance analysis, informed by 19 expert elicitations, then ranks the internal self-consistency of approximately 1.5 million combinations of trends. Based on the 1000 most consistent combinations, they conclude that high trends for innovation capacity could lower challenges to mitigation but not necessarily challenges to adaptation. Further, they find that a low trend for quality of governance consistently corresponds to higher challenges to adaptation. Additional research on innovation capacity and governance may improve the characterization of challenges to mitigation or adaptation.

The third paper by Lane and Montgomery (2013) argues that more explicit consideration of insights from the political science and political economy literatures could enhance the consistency and empirical realism of any new scenarios. The authors hail from the school of "rational choice institutionalism" (RCI), a field of social science that examines how actors work within and use institutions to achieve their goals. The authors show how RCI might shed light on the possibilities for economic growth, and for mitigation and adaptation policies in states with different types of governance. The modeling techniques currently used to generate the RCPs do not consider such institutional effects. Accounting for institutions via RCI and related literatures could change estimated net benefits of reduced radiative forcing and could influence evaluations of the appropriate balances between mitigation and adaptation. It can also help to more fully and consistently link the pathways with their underlying socioeconomic (in particular institutional) assumptions. Including scholars who study institutions and their effects on socio-economic development in the SSP process could better ground future scenarios in the theory and historical evidence about the determinants of economic growth and could enrich the ability of the SPAs to encompass a range of less-than-optimal policies consistent with underlying institutional assumptions.

The third group of three papers deals with ways of rendering the new scenarios more useful to different user communities through pattern-scaling as a way to provide place-specific information about RCPs and SSPs and to enhance the relevance of the scenarios for the impacts, vulnerability and adaptation research communities.

The first paper by Tebaldi and Arblaster 2013 reviews the ideas behind the pattern scaling technique, and focus on its value and limitations in view of its use for impact assessment and within integrated assessment models. The method of pattern scaling as a way of determining regionally differentiated projections of future climate change for intermediate radiative forcings for which simulations from fully coupled global climate models (GCMs) are not available. Such projections would be used for impact assessment, especially useful when considering the effects of mitigation policies for which explicit simulations are not available by construction, as the policy measures would be modeled as a result of reactive behavior to RCP outcomes. They present estimates of patterns for temperature and precipitation change from the latest transient runs based on RCPs, characterize the sources of variability across models and scenarios, and compare them to those obtained from the previous set of experiments. 
The second paper by Wilbanks and Ebi (2013) argue that climate change impact, adaptation and vulnerability (IAV) research communities need the information about how factors that determine impacts change in the scenarios and yet the SSPs are framed mainly in terms of mitigation and adaptation capacities. Research by the IAV community over the past decade is highlighting the complexity of factors and their interactions that determine impacts, indicating that descriptions of future development pathways must consider traditional socioeconomic dimensions such as economic growth, demographic change, changing land use, and technology development - but also other dimensions, such as institutional change. For IAV researchers, developing characterizations of alternative socioeconomic pathways to accompany climate change scenarios has historically been undermined by the lack of projections of long-term economic and social trends, especially at a level of detail appropriate to regional and more localized analysis.

In the third paper, van Ruijven et al. (2013) also discuss the role and relevance of a new SSP framework for IAV research. They first provide an overview of uses of socioeconomic scenarios in IAV studies and identifies the main shortcomings of earlier global environmental scenarios for IAV analyses. Second, the paper elaborates on two aspects of the SSPs that need to be improved in order to enhance their usefulness for IAV studies: the ability to work coherently across spatial scales and include indicators for assessing future vulnerability and adaptive capacity. In addition to standard scenario indicators of population and gross domestic product, this paper presents a research agenda to add income distribution, spatial population, health projections and governance indicators to the SSPs.

The last two papers present new concepts for the generation of new scenarios. The first by Rothman et al. (2013) presents a number of implications for the operationalization of the concept in the basic and extended versions of the SSPs. First, the elements comprising challenges to adaptation must include a wide range of socioeconomic and even some (non-climatic) biophysical factors. Second, careful consideration must be given to differences in these factors across scales, as well as cross-scale interactions. Third, any representation of the concept will require both quantitative and qualitative elements. The scenario framework offers the opportunity for the SSPs and full scenarios to be of greater value than has been the case in past exercises to both IAM and IAV researcher communities, but this will require a renegotiation of the traditional, primarily unidirectional relationship between the two communities.

The last paper, by Julie Rozenberg, Céline Guivarch and others, proposes a new concept to develop SSPs using a "backward" approach. The methodology first identifies a priori a large number of potential drivers of mitigation and adaptation challenges; uses an IAM to explore many thousands of combinations of these drivers thereby creating a large database of scenarios; then uses statistical cluster-finding algorithms to select a posteriori a few SSPs among these scenarios along with the most important drivers of the challenges to adaptation and mitigation. This methodology is applied to the selection of a few SSPs, but it could also be applied to any specific decisions faced by decision-makers. From a large database of runs built by many models, the methodology would allow selecting the most relevant scenarios for a specific decision, i.e. scenarios that best distinguish those future conditions in which proposed policy performs poorly from those in which it performs well.

Together, these essays and papers offer a rich and varied view of these new socioeconomic scenarios and the innovative process that has produced them, some initial indications of how they might be used, and initial suggestions on how the process might evolve into the future to derive full scenarios combining SSPs, SPAs and RCPs. The decision- support literature emphasizes that the process of developing policy-relevant information - that is the ways in which providers and users interact to produce information - is at least as important as the information products themselves (NRC 2009). This is particularly true with scenarios and the processes that create them (Parson et al. 2007). It is worth noting then that this special issue 
provides only a temporal snapshot of what is sure to be a long-term activity of developing new socioeconomic scenarios. We close by highlighting some future directions that in our view emerge from this special issue and the process that produced it.

Future research may delve deeper into the implications of the blending together of two distinct scenario traditions - narrative and quantitative - in this new SSP process. Narrative scenarios, sometimes characterized as storylines, provide rich contexts for understanding main drivers of change and resulting future outcomes. The narratives can be interpreted for many different spatial and temporal scales, and require a succinct logic. But they can be difficult to evaluate in part because they are so integrative in nature, and one cannot be sure that they have in fact spanned the potential universe of outcomes, thus making an assessment of their internal consistency or likelihoods quite difficult. The quantitative scenario tradition is model based and uses numeric assumptions to determine alternative future outcomes. These scenarios are essential for determining time paths and dynamics of future changes. But the assumptions about socioeconomic pathways are embedded in both the structure of the individual models and in the judgment of the modeling teams, and the models are by definition incomplete descriptions of the world, making it difficult to match them with the broader context. The IAV community has used both approaches, but many of their studies have dealt with local and regional changes, and the relationship of such local/regional scenarios to the more global scenario traditions is at best unclear. The degree to which it is reasonable to connect the spatial and temporal scales of both traditions so as to have a more complete description of potential futures is still very much a potential research topic.

Both traditions have other advantages and shortcomings. IPCC SRES scenarios first integrated the two approaches. Four narrative storylines were first developed through an elaborate deliberative process. The time consuming process had to be completed before climate change simulations could be run. Only then could impacts, adaptation and vulnerability assessment could be conducted, a process that is still going on over a decade later. The RCPs, SSPs and SPAs processes aim to shorten this process significantly by undertaking climate change simulations based on RCPs while integrated assessment models in parallel develop narrative storylines and SSPs and SPAs quantifications consistent with RCPs. But this process raises questions beyond those in this special issue. For instance, how might the SSPs and RCPs need to change as an outcome of this integration. Is there a small number of succinct storylines that can encompass the "library" of quantitative (and narrative) scenarios that emerge out the RCP, SSP and SPA process? How could the rates of change and dynamics of SSPs be related to plausible narratives of behavioral and lifestyle changes that are implied by low or high-level stabilization RCPs? Is there a way of combining different regional quantifications, say from different SSPs, and what would corresponding narratives look like? To what degree to regionally specific scenarios need to be consistent with global SSPs?

Ebi et al. (2013) echo these themes in their call for future research on determining the relevance of the SSP narratives for different problems, scales and research questions and on examining how to relate scenarios on global and local scales. Future research may also need to continue the work begun by van Vuuren et al. (2013) by mapping previous scenarios onto the current SSP's, and the current SSP's onto those that follow them.

The current framework and scenarios will also hopefully serve many research and decisionmaking needs. One might reasonably ask questions related to other societal goals, e.g. food security or access to water resources, and use future scenario processes to address whether or not change in the climate system, or climate policy measures will make these goals more difficult or easier to meet. Future work will be needed to examine how these current products might be augmented to address additional questions. Ebi et al. (2013) also call for future research to distinguish the questions the current SSP can and cannot address and to 
integrated the drivers of development into the SSP narratives, since climate policy is increasingly considered as one aspect of development.

As a potential longer-term agenda, the Boulder meeting in Fall 2011 also envisioned a concept one might call "scenarios on demand." The current SSP process envisions providing a product: a small number of global scenarios that can help coordinate the work of numerous researchers as well as provide planning tools for numerous decision makers. In the future, one might imagine providing a service (Parson et al. 2007; Lempert 2012): a vast array of data and model runs along with web-based tools, perhaps along the lines of those suggested here by Rozenberg et al. (2013) and Schweizer and O'Neill (2013), that would allow users to construct a set of well-informed, selfconsistent scenarios customized for their particular application. Ebi et al. (2013) similarly suggests exploiting large databases of scenarios and considering wild cards or surprising scenarios. More broadly, such a "scenarios on demand" concept might represent one instance of a new blending of the narrative and quantitative scenario traditions by using quantitative data and analysis to facilitate the process of choosing scenarios and suggesting which storylines are most important to develop.

Finally, the community may find it useful to establish an explicit process that balances the need for continuity with the need for customization and for innovation as more people come to use these new scenarios. The SRES scenarios remain in broad use after more than a decade, and have been taken up by a broad swath of research communities with interests that range from climate change to biodiversity conservation. However, there has been no formal process to include users' insights and needs back into the scenarios themselves after they have been completed even though many of IAMs that developed the original scenarios have offered updates and revisions periodically. The new process, while more inclusive from a disciplinary perspective at its outset, has not yet been used by many investigators nor involved a sufficiently wide range of people. The continued development of new SSP's, new RCP's, or new socioeconomic policy assumptions would clearly benefit from even broader engagement and involvement of both more and of a wider variety of researchers with different interests and perspectives. Macroeconomists, land-use planners, conservation biologists, and scholars of urban issues, to name just a few, could all potentially add interesting perspectives on the effort. One key is to establish a process that allows and encourages such ongoing input, while at the same time providing a sufficiently stable set of scenarios to enable collaboration among the diverse climate change research communities.

It has been over a decade since the last set of socioeconomic scenarios was developed. The number of potential users of these new SSP's is already much larger than that when SRES was new. In the future, the number and diversity of users is likely to grow larger still. This new framework and scenarios represent a major milestone, one that will hopefully lay a foundation for important research and climate-related decision making long into the future.

Acknowledgments The authors would like to thank the anonymous reviewers for their valuable comments and suggestions that have improved this introductory paper and acknowledge with gratitude the contributions of other authors to this Special Issue on which this introductory paper is based.

Open Access This article is distributed under the terms of the Creative Commons Attribution License which permits any use, distribution, and reproduction in any medium, provided the original author(s) and the source are credited.

\section{References}

Arnell N, Kram T, Carter T, Ebi K, Edmonds J, Hallegatte S, Kriegler E, Mathur R, O'Neill BC, Riahi K, Winkler H, van Vuuren D, Zwickel T (2011) A framework for a new generation of socioeconomic scenarios for climate change impact, adaptation, vulnerability and mitigation research. Scenario Framework Paper 
IPCC (1990) Climate change: the scientific assessment. In: Houghton, JT, Jenkins GJ, Ephraums JJ (eds) Report prepared for Intergovernmental Panel on Climate Change by Working Group I. Cambridge University Press, Cambridge, Great Britain, New York, USA and Melbourne, Australia

IPCC (2012) Workshop report of the intergovernmental panel on climate change workshop on socio-economic scenarios. In: Edenhofer O, Pichs-Madruga R, Sokona Y, Barros V, Field CB, Zwickel T, Schloemer S, Ebi $\mathrm{K}$, Mastrandrea M, Mach K, von Stechow C (eds) IPCC working group III technical support unit. Potsdam Institute for Climate Impact Research, Potsdam, p 51

Kriegler E, O'Neill BC, Hallegatte S, Kram T, Lempert R, Moss R, Wilbanks T (2012) The need for and use of socio-economic scenarios for climate change analysis: a new approach based on shared socio-economic pathways. Glob Environ Chang 22(4):807-822

Leggett J, Pepper WJ, Swart RJ (1992) Emissions scenarios for IPCC. In: Houghton JT, Callander BA, Varney SK (eds) An Update. In Climate Change 1992. The supplementary report to the IPCC Scientific Assessment, Cambridge University Press, Cambridge, UK, pp. 69-95

Lempert RJ (2012) "Climate scenarios that illuminate vulnerabilities and robust responses." Climatic Change

Meehl GA, Hibbard K (2007) A strategy for climate change stabilization experiments with AOGCMs and ESMs. Report from the Aspen Global Change Institute 2006 Session - Earth system models: the next generation, July 30 - August 5, 2006 and joint WGCM/AIMES steering committee meeting 27 September 2006, WCRP Informal Report No 3/2007; ICPO Publication No. 112; IGBP Report No. 57

Moss RH, Babiker M, Brinkman S, Calvo E, Carter T, Edmonds J, Elgizouli I, Emori S, Erda L, Hibbard K, Jones R, Kainuma M, Kelleher J, Lamarque JF, Manning M, Matthews B, Meehl J, Meyer L, Mitchell J, Nakićenović N, O’Neill B, Pichs R, Riahi K, Rose S, Runci P, Stouffer R, van Vuuren D, Weyant J, Wilbanks T, van Ypersele JP, Zurek M (2008) Towards new scenarios for analysis of emissions, climate change, impacts, and response strategies. Intergovernmental Panel on Climate Change (IPCC), Geneva

Moss RH, Edmonds JA et al (2010) The next generation of scenarios for climate change research and assessment. Nature 463(7282):747-756

Nakicenovic N, Alcamo J et al (2000) Special report on emissions scenarios. Working Group III of the intergovernmental panel on climate change, IPCC. Cambridge University Press, Cambridge

NRC (2009). Informing decisions in a changing climate. T. N. A. Press, Washington, DC, Panel on strategies and methods for climate-related decision support, committee on the human dimensions of climate change, division of behavioral and social sciences and education. National Research Council (NRC)

NRC (2010) Panel on socioeconomic scenarios for climate change research and assessment, describing socioeconomic futures for climate change research and assessment: Report of a Workshop, National Research Council (NRC)

O'Neill BC, Carter TR, et al. (2012) "Meeting report of the workshop on the nature and use of new socioeconomic pathways for climate change research, Boulder, CO, November 2-4, 2011"

Parson EA, Burkett V, et al. (2007) Global-change scenarios: Their development and use, synthesis and assessment product 2.1b, US Climate Change Science Program

Pepper WJ, Leggett J, Swart R, Wasson J, Edmonds J, Mintzer I, (1992) Emissions scenarios for the IPCC. In: Houghton JT, Callandar BA, Varney SK (eds) An update: assumptions, methodology, and results: Support Document for Chapter A3. In Climate Change 1992: Supplementary Report to the IPCC Scientific Assessment, Cambridge University Press, Cambridge

Taylor KE, Stouffer RJ, Meehl GA (2012) An overview of CMIP5 and the experiment design. Bull Amer Meteor Soc 93:485-498. doi:10.1175/BAMS-D-11-00094.1

van Vuuren DP, Edmonds JA et al (2011) A special issue on the RCPs. Clim Chang 109(1-2):1-4

van Vuuren DP, Riahi K et al (2012) A proposal for a new scenario framework to support research and assessment in different climate research communities. Glob Environ Chang 22(1):21-35

\section{Papers in this issue}

Ebi K, O’Neill B, et al. (2013) A new scenario framework for climate change research: background, process, and future directions. Climatic Change Special Issue. doi:10.1007/s10584-013-0912-3

Kriegler E, Edmonds J, Hallegatte S, Ebi K, Kram T, Riahi K, Winkler H, van Vuuren DP (2013) A new scenario framework for climate change research: the concept of shared climate policy assumptions. Climatic Change Special Issue (this issue)

Lane L, Montgomery WD (2013) An institutional critique of new climate scenarios. Climatic Change Special Issue. doi:10.1007/s10584-013-0919-9

O’Neill B, Kriegler E, Riahi K, Ebi K, Hallegatte S, Carter T, Mathur R, van Vuuren DP (2013) A new scenario framework for Climate Change Research: the concept of Shared Socio economic Pathways. Climatic Change Special Issue. doi:10.1007/s10584-013-0919-9 
Rothman DS, Romero-Lankao P, Schweizer V, Bee BA (2013) Challenges to adaptation: a fundamental concept for a new generation of socioeconomic scenarios. Climatic Change Special Issue. doi:10.1007/s10584-0130907-0X

Rozenberg J, Guivarch C, Lempert R, Hallegatte S (2013) Building SSPs for climate policy analysis: a scenario elicitation methodology to map the space of possible future challenges to mitigation and adaptation. Climatic Change Special Issue. doi:10.1007/s10584-013-0904-3

Schweizer VJ, O'Neill B (2013) Systematic construction of global socioeconomic pathways using internally consistent element combinations. Climatic Change Special Issue. doi:10.1007/s10584-013-0908-z

Tebaldi C, Arblaster J (2013) Pattern scaling: a review of its strengths and limitations, and an update on the latest model simulations. Climatic Change Special Issue. doi:10.1007/s10584-013-1032-9

van Ruijven BJ, Levy M, Agrawal A, Biermann F, Birkmann J, Carter TR, Ebi K, Jones B, Jones R, KempBenedict E, Kok M, Kok K, Lemos MA, Lucas PL, Orlove B, Pachauri S, Parris T, Patwardhan A, Peterson A, Preston BL, Ribot J, Rothman DS, Schweizer VJ (2013) Enhancing the relevance of global shared socioeconomic pathways for climate change impacts, vulnerability and adaptation research. Climatic Change Special Issue. doi:10.1007/s10584-013-0931-0

van Vuuren DP, Kriegler E, O’Neill B, Ebi K, Riahi K, Carter TR, Edmonds J, Hallegatte S, Kram T, Mathur R, Winkler H (2013) A new scenario framework for Climate Change Research: scenario matrix architecture. Climatic Change Special Issue. doi:10.1007/s10584-013-0906-1

van Vuuren DP, Carter T (2013) Mapping of past scenarios to the SSP framework. Climatic Change Special Issue. doi:10.1007/s10584-013-0974-2

Wilbanks TJ, Ebi K (2013) SSPs from an impact and adaptation perspective. Climatic Change Special Issue. doi: 10.1007/s10584-013-0903-4 